\title{
EPILEPSY
}

\section{New insights into the treatment and consequences of poststroke epilepsy}

$\mathrm{R}$ esearch conducted in Taiwan and reported in the European Journal of Neurology suggests that newgeneration antiepileptic drugs provide more-effective seizure control than some traditional antiepileptic drugs in patients with poststroke epilepsy. The importance of exploring the best treatment options for this condition was further underlined by a separate study from the Netherlands, which identified a heightened risk of mortality in young patients who developed epilepsy following a stroke.

"As the Taiwanese population is on its course from an 'ageing' society to an 'aged' society, incident cases of stroke-and, consequently, the numbers of patients with poststroke epilepsy-are expected to increase," explains Li-Nien Chien, the corresponding author for the Taiwanese study. "The lack of clear therapeutic guidelines leaves physicians to face the dilemma of which would be the most appropriate antiepileptic drug for the management of poststroke epilepsy."

Using a retrospective observational study design, Chien and colleagues assessed the frequency of emergency room visits and subsequent hospitalizations for recurrent seizures in 3,622 patients with late-onset poststroke epilepsy (defined by the authors as seizure onset more than 14 days after stroke). The participants were stratified according to whether they had been prescribed phenytoin, valproic acid, carbemazepine or new-generation antiepileptic drugs. The latter category consisted of oxcarbazepine, vigabatrin, tiagabine, lamotrigine, topiramate, gabapentin, levetiracetam and pregabalin, none of which were prescribed at a sufficient frequency to be analysed on their own.

Despite being the most frequently prescribed drug for poststroke epilepsy in the study population, phenytoin carried the highest risk of seizure recurrence. By contrast, the new-generation antiepileptic drugs, which were rarely prescribed for this indication, produced the most favourable outcomes in terms of the incidence of emergency room visits and hospitalizations. The results indicate that the newer antiepileptic drugs warrant further investigation for the management of patients with poststroke epilepsy.

"We hope that our study will assist clinicians in choosing the most appropriate antiepileptic drug for their patients with poststroke epilepsy," says Chien. "Since our research is the result of a study based on a single population, we look forward to seeing future studies in other populations, as well studies with a higher level of evidence, such as randomized controlled trials."

The Dutch investigation, which formed part of the FUTURE (Follow-Up of Transient Ischemic Attack and Stroke Patients and Unelucidated Risk Factor Evaluation) study, measured all-cause case fatality (death within 30 days after the index event) and long-term mortality in patients aged 18-50 years with firstever stroke or transient ischaemic attack. Of a total of 631 participants, 76 (12\%) developed poststroke epilepsy.

"The FUTURE study is a prospective cohort study on the causes and longterm consequences of stroke at a young age," explains Frank-Erik de Leeuw, who coordinated the study. "So far, there have been no studies in the young on the association between poststroke epilepsy and mortality."

The researchers reported that poststroke epilepsy was associated with significant increases in both case fatality and long-term mortality (HRs 4.8 and 1.8 , respectively) in young patients with stroke. The relationship was particularly strong in patients with mild stroke, perhaps indicating that factors other than poststroke epilepsy become more important contributors to mortality as stroke severity increases.

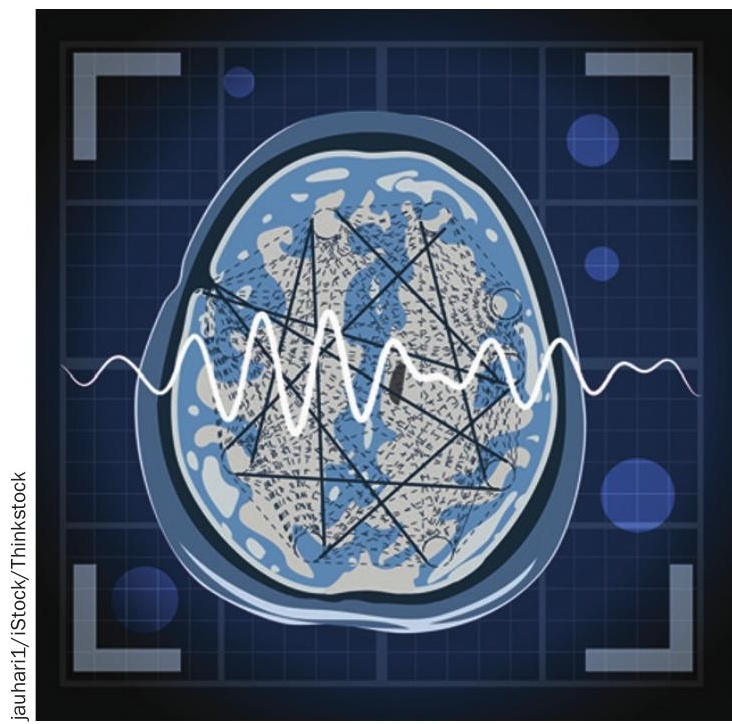

"Poststroke epilepsy is a common problem after stroke at a young age, and is associated with serious complications such as a poorer functional outcome and high mortality," concludes de Leeuw.

The FUTURE investigators point out that the next logical step would be to explore whether prophylactic antiepileptic drugs use can improve outcomes after stroke in the younger population. In a similar vein, Chien and colleagues are now trying to identify prognostic factors for the development of poststroke epilepsy. These biomarkers might help pinpoint individuals who could benefit from early initiation of treatment with antiepileptic drugs after stroke.

Heather Wood

Original articles Huang, Y.-H. et al. Efficacy of phenytoin, valproic acid, carbamazepine and new antiepileptic drugs on control of late-onset post-stroke epilepsy in Taiwan. Eur. J. Neurol. doi:10.1111/ene.12766 | Arntz, R. M. et al. Poststroke epilepsy is associated with a high mortality after a stroke at young age: Follow-Up of Transient Ischemic Attack and Stroke Patients and Unelucidated Risk Factor Evaluation study. Stroke doi:10.1161/ STROKEAHA.115.010115 